\title{
Insights from the protein-protein interaction network analysis of Mycobacterium tuberculosis toxin- antitoxin systems
}

\author{
Zoozeal Thakur1, Renu Dharra ${ }^{1}$, Vandana Saini ${ }^{2,}$ Ajit Kumar ${ }^{*}$, Promod K. Mehta $^{1^{*}}$ \\ ${ }^{1}$ Centre for Biotechnology, Maharshi Dayanand University (MDU), Rohtak-124001 (Haryana), India; ${ }^{2}$ Toxicology \& Computational \\ Biology Group, Centre for Bioinformatics, Maharshi Dayanand University (MDU), Rohtak-124001 (Haryana), India; Promod K. Mehta \\ - E-mail: pkmehta3@hotmail.com; Ajit Kumar - E-mail: akumar.acbt.mdu@gmail.com; Telephone No. +91- 9896504193; +91- \\ 9802299176; *Corresponding authors
}

Received October 6, 2017; Revised November 11, 2017; Accepted November 11, 2017; Published November 30, 2017

\begin{abstract}
:
Protein-protein interaction (PPI) network analysis is a powerful strategy to understand M. tuberculosis $(M t b)$ system level physiology in the identification of hub proteins. In the present study, the PPI network of $79 \mathrm{Mtb}$ toxin-antitoxin (TA) systems comprising of 167 nodes and 234 edges was investigated. The topological properties of PPI network were examined by 'Network analyzer' a cytoscape plugin app and STRING database. The key enriched biological processes and the molecular functions of Mtb TA systems were analyzed by STRING. Manual curation of the PPI data identified four proteins (i.e. Rv2762c, VapB14, VapB42 and VapC42) to possess the highest number of interacting partners. The top $15 \%$ hub proteins were identified in the PPI network by employing two statistical measures, i.e. betweenness and radiality by employing cytohubba. Insights gained from the molecular protein models of VapC9 and VapC10 are also documented.
\end{abstract}

Keywords: Mycobacterium tuberculosis, Toxin-antitoxin, STRING, Cytoscape, Homology Modeling

Abbreviations: Toxin-antitoxin, TA; Mycobacterium tuberculosis, Mtb; Gene ontology, GO; Protein-protein interaction, PPI.

\section{Background:}

The ability of Mycobacterium tuberculosis $(M t b)$ to persist inside the host cells under a variety of adverse conditions including oxidative stress, nutrient starvation and hypoxia helps to understand pathogenesis [1, 2]. Mtb toxin-antitoxin (TA) systems comprising of two component genetic modules - a stable toxin and relatively unstable antitoxin, play a significant role for the survival of bacteria under stress conditions. $M t b$ harbors a high number of TA systems (79) belonging to various families such as VapBC, MazEF, ParDE, higBA, RelBE, and several uncharacterized TA systems [3]. These TA systems are associated with antibiotic resistance, biofilm formation and persistence inside the host cells [4]. In response to stress conditions, the labile antitoxin is degraded and toxin is released, which in turn halts transcription, translation etc. and that leads to growth inhibition and even cell death [3].
Protein-protein interaction (PPI) is imperative to many cellular process including signal transduction, transcriptional regulation, post-translational modification, etc. [5]. PPI network analysis is a robust approach to understand the mechanisms associated with mycobacterial pathogenesis, functional annotation of genes, etc. $[6,7]$ PPIs can be detected by computational and experimental methods. Experimental methods include yeast two-hybrid system, tandem affinity purification and protein microarrays, whereas computational methods include interlog-based method and prediction based on genetic algorithms. In comparison to experimental techniques, computational methods take less time and are inexpensive [8]. In the present study, we report the topological and functional enrichment analysis of PPI network of $79 M+b$ TA systems constructed from STRING v10.5 and Cytoscape v3.5.0. The molecular models of VapC9 and VapC10 have also been documented to gain functional insights. 


\section{Open access}

Methodology:

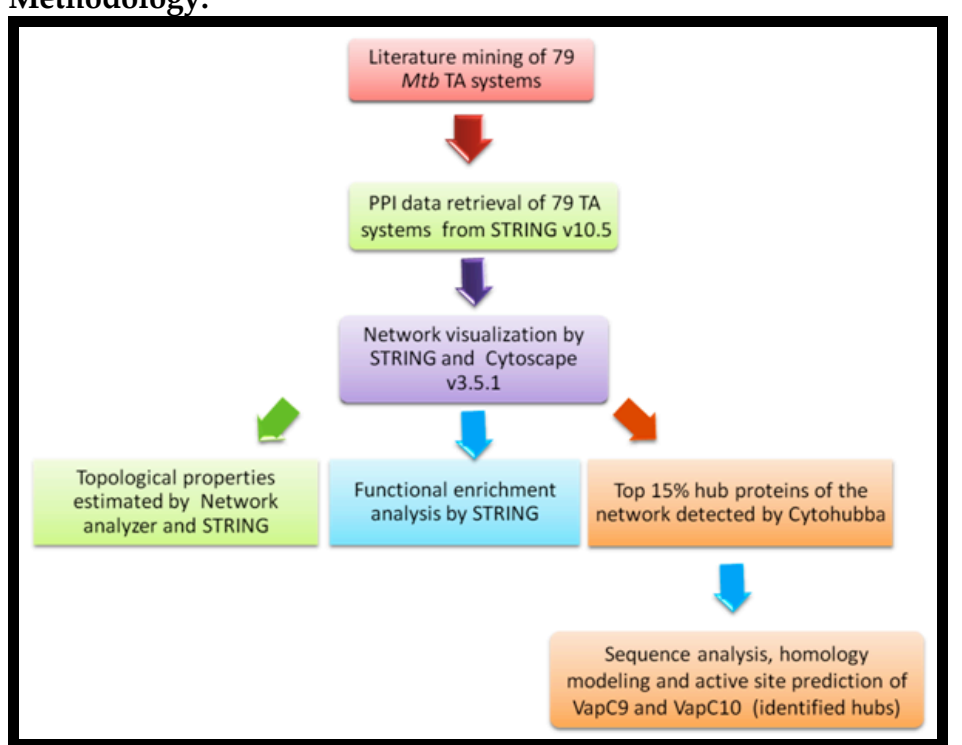

Figure 1: A flowchart representing the methodology applied in the study; arrows represent flow of information and transition from one step to another

\section{Literature mining of $M t b$ TA system genes:}

A total of $79 \mathrm{M} t b \mathrm{H}_{37} \mathrm{Rv}$ TA system genes enlisted in Suppl Table 1 were mined from the literature [3]. The mined 79 TA systems belonged to various TA families including VapBC (50 members), MazEF (10 members), HigBA (3), ParDE (2), RelBE (2), YefM/YoeB (1) and 11 unclassified TA system genes Figure 1.

\section{PPI Network construction:}

The candidate $79 \mathrm{Mtb}$ TA systems were converted into seed sequences to mine PPI data from STRING (Search tool for the Retrieval of Interacting Genes/Proteins) v10.5 database (http://string-db.org) [9]. Interaction sources selected for generation of PPI network were text mining, experiments, databases, co-expression, neighborhood, gene fusion, and cooccurrence. PPIs that possessed at least a medium confidence score of 0.400 were considered for network generation. Network construction and visualization was done by cytoscape v 3.5.1 [10] and STRING v10.5.

\section{Topological and functional enrichment analysis of PPI} network:

The topological parameters of PPI network, i.e. number of nodes, number of edges, average node degree, etc were evaluated by STRING v10.5. The protein-protein association data obtained from STRING was further utilized to compute several other topological parameters such as average clustering coefficients, topological coefficients, and shortest path lengths etc. via Network analyzer, a cytoscape plugin app, by treating the network as directed graph. In addition, functional enrichment of input seed sequences of $M t b$ TA systems was carried out by STRING to identify significantly enriched GO (Gene Ontology) biological processes and molecular functions.

\section{Identification of $\mathrm{Hub}$ proteins:}

Cyto-Hubba [11], a java plugin for Cytoscape software, was employed to determine the hub proteins of PPI network of $M t b$ TA systems. In this study, two centrality measurements, i.e. betweenness and radiality were applied to mine the top $15 \%$ hub proteins of the network. In addition, we further mined hub proteins, which were commonly identified by both the algorithms.

\section{Sequence analysis of VapC9 and VapC10:}

Protein sequence information of VapC9 and VapC10 was retrieved from Tuberculist database [12]. Various domain identification tools including InterProScan [13], Pfam [14], and NCBI CDD (Conserved domain database), [15] were employed to detect the conserved domains present in the protein sequences, which in turn carried out sequence similarity search with the close orthologus family members. The physical and chemical properties such as extinction coefficient, instability index, and aliphatic index, GRAVY etc. were determined by Protaparam tool of ExPASy $[15,16]$.

\section{Homology modeling of VapC9 and VapC10:}

BLASTp search with default parameters was carried out against PDB database to identify the suitable templates for construction of homology models of VapC9 and VapC10. The search identified 2FE1 (resolution $2.2 \AA$ ) and 2H1C (resolution $1.8 \AA$ ) as the best suitable template structures for VapC 9 and VapC10, respectively. Modeler v9.17 was utilized for the construction of homology model of VapC9 and VapC10. The energy minimization of constructed 3D models was performed by chimera v1.11.2.

\section{Model evaluation:}

Energy minimized 3D models of VapC9 and VapC10 were subjected to various model validation servers to evaluate the stereo chemical properties. To determine parameters such as Zscore, QMean Score, D-fire Energy and residue by residue geometry, energy minimized theoretical models were subjected to SWISS-MODEL server [17]. Various other model evaluation tools were also employed, i.e. ERRAT [18], ProQ [19], Molprobity [20], RESPROX [21] and ProSA-web [22] to determine the model quality.

\section{Active site prediction:}

Metapocket 2.0 was employed to determine active site of VapC9 and VapC10 [23]. Metapocket uses consensus approach to detect ligand-binding sites by employing eight methods: LIGSITE, PASS, SURFNET, Fpocket, GHECOM, ConCavity, POC ASA and Q-Sitefinder. 


\section{Open access}

Results and Discussion:

\section{PPI network analysis of $M t b$ TA systems:}

Protein-protein association data of 79 TA systems of $M t b \mathrm{H}_{37} \mathrm{Rv}$ was extracted from STRING v 10.5. To completely explore the PPI data, the search was set to include all the source parameters. The mined PPI data was comprised of total 468 PPI's as depicted in Figure 2. Manual curation of the PPI data revealed that 63 proteins out of 468 PPIs possessed $\geq 4$ interacting partners in the network (Suppl Table 2), whereas 64 proteins were associated with only one interacting partner. Two groups of proteins, comprising of 20 and 15 proteins were highly connected with each member having five and six interacting partners, respectively. Notably, two members of VapBC family, i.e. antitoxin VapB45 and antitoxin VapB14 were found to possess 8 interacting partners each. Interestingly, both the toxin and antitoxin of VapBC42 were highly connected with each possessing 9 interacting partners. Strikingly, antidote HigA1 possessed the highest number (10) of interacting partners. For 17 proteins, no PPI information could be extracted from STRING. PPI information extracted from STRING v10.5 ranged from medium to highest confidence scores. In fact, $84(\sim 18 \%)$ of the protein-protein associations fell within highest confidence interval (CI, S > 0.9), 176 (37.6\%) within high $\mathrm{CI}(0.7 \leq \mathrm{S}<0.9)$, and $208(44.44 \%)$ within medium CI $(0.4 \leq \mathrm{S}<0.7)$.

In addition, topological and functional enrichment analysis of input $M t b$ TA systems was carried out by STRING and 'network analyzer' a cytoscape plugin. Network statistics obtained by STRING database revealed that the extracted interactome was comprised of 167 nodes and 234 edges. The average node degree and average local clustering coefficient of the network was determined to be 2.8 and 0.628 , respectively (Table1). On the other hand, 'network analyzer' a cytoscape plugin estimated several other topological parameters such as network diameter, network radius, shortest path, characteristic path length and average number of neighbors (Table 1). GO biological process and molecular function enrichment analysis of input seed sequences was carried out by STRING v10.5. The majority of $M t b$ TA system proteins were significantly enriched in biological processes associated with regulation of growth (1.46E-77), nucleic acid phosphodiester bond hydrolysis (1.08E-61), RNA phosphodiester bond hydrolysis (3.73E-60) and negative regulation of growth (2.65e-45, Table 2). Furthermore, molecular functions of such proteins were primarily related with nuclease activity (6.27e-62), ribonuclease activity (7.72e-61), and metal ion binding (7.36e-12, Table 3). Similar to gene enrichment analysis of this study, activation of TA systems leading to growth arrest by the toxin partners of VapBC, RelBE, MazEF, and HigBA families has also been reported [3].

Hub proteins of PPI network represent highly connected nodes with special biological properties and are more evolutionary conserved than non-hubs. In fact, removal of such hubs can lead to network disruption and thus are considered as attractive drug targets [24, 25]. Identification of hub proteins can be carried out by in silico tools such as Hubba, cytohubba, and CHAT etc. [26, 27]. In the present study, cytohubba was used to explore the hub proteins of $M t b$ TA systems PPI network. The top 15\% hub proteins were identified on the basis of radiality and betweenness algorithms (Table 3, 4). HigA1 and VapB45 antitoxins were determined to be the top scorer hub proteins by betweenness and radiality method, respectively. Antitoxin HigA1 (Rv1956) belonging to HigBA family has been reported among the 10 top most upregulated $M t b$ TA systems of drug tolerant persisters [3]. On the other hand, VapB45 (Rv2018) is the antitoxin partner of VapC45, but no experimental data is available to elucidate their role in $M t b$ pathogenesis. In addition, we mined the hub proteins that were commonly identified by both the algorithms (Table 4). Notably, majority of the hub proteins identified belonged to the VapBC family apart from the members of HigBA and RelBE families. VapBC is the largest family of $M t b$ TA systems characterized by the presence of PIN domain and functions mostly as ribonucleases [3, 28]. Interestingly, Rv2762, which was not part of input seed proteins, was also detected as a top ranker hub protein.

Antitoxins are reported to be small proteins with less order in their structure. Therefore, it is difficult to find druggable pockets on their surface that can accommodate small-molecule inhibitors, whereas toxins are more stable and ordered in their structure, and are also considered as attractive targets for drug-design [28]. Therefore, we focused to elucidate the structural insight of toxins identified by in silico analysis. It was found that 5 out of 10 top ranking hub proteins were antitoxins, i.e. higA1, VapB45, VapB14, VapB11 and RelB, whereas three proteins were toxins, i.e. VapC1, VapC9 and VapC10. Since the structure of VapBC1 is available at $\mathrm{PDB}$, we focused to determine the structural insights of VapC9 and VapC10 proteins. Domain identification tools used in the study, i.e NCBI CDD, Pfam and InterProscan revealed the presence of PIN domain in both VapC9 and VapC10. In fact, PIN domains are small protein domains of $\sim 130$ amino acids, which are characterized by the presence of three invariant amino acid residues and fourth lesser-conserved acidic residue [3, 29]. Physicochemical properties were computed by protparam tool of ExPASy for VapC9 and VapC10 (Table 5). Protein BLAST was carried out against PDB database to identify homologs with resolved 3D structure for structure prediction of VapC9 and VapC10. The crystal structure of PAE0151 from Pyrobaculum aerophilum (2FE1) was selected as the best suitable template on the basis of maximum query coverage $(100 \%)$ and maximum identity $(79 \%)$ for input VapC9 protein sequence (Figure 3). In a similar manner, crystal Structure of Fitacb from Neisseria gonorrhoeae (2H1C) was chosen as the best template structure for the development of homology model of VapC10 based on maximum sequence coverage $(81 \%)$ and maximum sequence identity (32\%) (Figure 4). Five theoretical models generated for each protein by Modeler v9.17 were assessed on the basis of 


\section{BIOINFORMATION}

\section{Discovery at the interface of physical and biological sciences}

\section{Open access}

DOPE score and GA341 score. The model with highest GA341 score and lowest DOPE score was selected and was further subjected to energy minimization by Chimera v1.11.2.

Theoretical models of VapC9 and VapC10 were subjected to various structural validation servers to assess the correctness of the models. Ramachandran plot obtained for VapC9 by PROCHECK of swiss model server revealed that $93 \%$ of amino acid residues were present in most favored regions, $6.1 \%$ in additionally allowed regions and $0.9 \%$ in generously allowed regions (Table 6). On the other hand, Ramachandran plot for VapC10 showed that $84.5 \%$ residues were present in the most favored region, $12.9 \%$ in additionally allowed region and $2.6 \%$ in generously allowed region (Table 6). Notably, for both the generated models, none of the amino acid residues was observed in the disallowed region. Overall G-factor score computed for VapC9 and VapC10 by swiss model server fell in the acceptable cut-off range. In addition, Z-score, Q-mean score and D-fire score were also estimated by swiss model server, which further confirmed the reliability of the models generated (Table 6). The overall quality factor score obtained after ERRAT analysis was $97.3 \%$ for VapC9 and $93.5 \%$ for VapC10. Prosa web Z score for VapC9 and VapC10 was -4.07 and -3.64 respectively, thereby suggesting that the structures are of good quality (Table 6). Predicted resolution by resprox along with LG and Maxsub score determined by ProQ also indicated the reliability of 3D models. In addition, MOLPROBITY revealed that none of the residue possessed bad bonds or $C \beta$ deviations $>0.25 \mathrm{~A}$ (Table 6). The 3D models generated in the study were of reliable quality as assessed by various structural assessment reports such as PROCHECK of swiss model server, ERRAT, ProSA-web, ProQ, MOLPROBITY and ResProx. In addition, active site of VapC9 and VapC10 was identified by Metapocket v2.0. The generated homology models and active site determined by metapocket v2.0 of VapC9 and VapC10, respectively are shown (Figure 5-6 A, B). The developed models can be used for structure based drug designing.

Table 1: Topological parameters of PPI network determined by STRING v10.5 and Network analyzer plugin of cytoscape 3.5.0.

\begin{tabular}{lll}
\hline SOURCE & NETWORK STATISTICS & \\
\hline STRING & Number of nodes & 167 \\
& Number of edges: & 234 \\
& Average node degree: & 2.8 \\
& Avg. local clustering coefficient & 0.628 \\
& Expected number of edges: & 41 \\
& PPI enrichment p-value & 0 \\
NETWORK ANALYZER & Number of nodes (excluding isolated nodes) & 157 \\
& Clustering coefficient & 0.137 \\
& Connected components & 25 \\
& Network diameter & 5 \\
& Network radius & 1 \\
& Shortest paths & 470 \\
& Characteristic path lengths & 1.787 \\
& Avg. number of neighbors & 2.981 \\
& Network density & 0.0 \\
& Isolated nodes & 0 \\
& Number of self loops & 0 \\
& Multi edge node pairs & 0. \\
\hline
\end{tabular}

Table 2: GO biological pathway enrichment analysis of PPI network for 79 MTb TA systems.

\begin{tabular}{llll}
\hline Pathway ID & Pathway description & Count in gene set & False discovery rate \\
\hline GO:0040008 & regulation of growth & 60 & $1.46 \mathrm{E}-77$ \\
GO:0090305 & nucleic acid phosphodiester bond hydrolysis & 61 & $1.08 \mathrm{E}-61$ \\
GO:0090501 & RNA phosphodiester bond hydrolysis & 49 & $3.73 \mathrm{E}-60$ \\
GO:0045926 & negative regulation of growth & 34 & $2.65 \mathrm{E}-45$ \\
GO:0045927 & positive regulation of growth & 31 & $2.75 \mathrm{E}-38$ \\
GO:0090304 & nucleic acid metabolic process & 67 & $2.59 \mathrm{E}-35$ \\
GO:0050789 & regulation of biological process & 64 & $1.60 \mathrm{E}-34$ \\
GO:0016070 & RNA metabolic process & 57 & $3.61 \mathrm{E}-33$ \\
GO:0048519 & negative regulation of biological process & 39 & $5.55 \mathrm{E}-31$ \\
GO:0048518 & positive regulation of biological process & 33 & $5.89 \mathrm{E}-28$ \\
GO:2000112 & regulation of cellular macromolecule biosynthetic process & 29 & $5.82 \mathrm{E}-14$ \\
GO:0010468 & regulation of gene expression & 29 & $2.04 \mathrm{E}-13$ \\
GO:0051171 & regulation of nitrogen compound metabolic process & 29 & $2.39 \mathrm{E}-13$ \\
GO:0080090 & regulation of primary metabolic process & 29 & $1.22 \mathrm{E}-12$ \\
GO:0031323 & regulation of cellular metabolic process & 29 & $1.69 \mathrm{E}-12$ \\
GO:0019222 & regulation of metabolic process & 30 & $1.74 \mathrm{E}-12$
\end{tabular}




\section{BIOINFORMATION}

\section{Discovery at the interf ace of physical and biological sciences}

\section{Open access}

GO:0051252

GO:0017148

GO:0006417

GO:0051172

GO:2000113

GO:0010605

GO:0010629

GO:0031324

GO:0009892

GO:0009987

GO:0006401

GO:0090502

GO:0008150

GO:0006402

GO:0016075

GO:0045727

GO:0051253 regulation of RNA metabolic process

negative regulation of translation

ulation of translation

negative regulation of nitrogen compound metabolic process

negative regulation of cellular macromolecule biosynthetic process

negative regulation of macromolecule metabolic process

negative regulation of gene expression

negative regulation of cellular metabolic process

negative regulation of metabolic process

ular process

RNA catabolic process

RNA phosphodiester bond hydrolysis, endonucleolytic

biological_process

mRNA catabolic process

rRNA catabolic process

positive regulation of translation

negative regulation of RNA metabolic process
$5.75 \mathrm{E}-09$

$9.06 \mathrm{E}-08$

$1.20 \mathrm{E}-07$

7.44E-07

$1.42 \mathrm{E}-06$

$3.98 \mathrm{E}-06$

$6.92 \mathrm{E}-06$

$9.52 \mathrm{E}-06$

$1.22 \mathrm{E}-05$

$2.21 \mathrm{E}-05$

$2.36 \mathrm{E}-05$

0.000136

0.000313

0.00466

0.00466

0.00466

0.0107

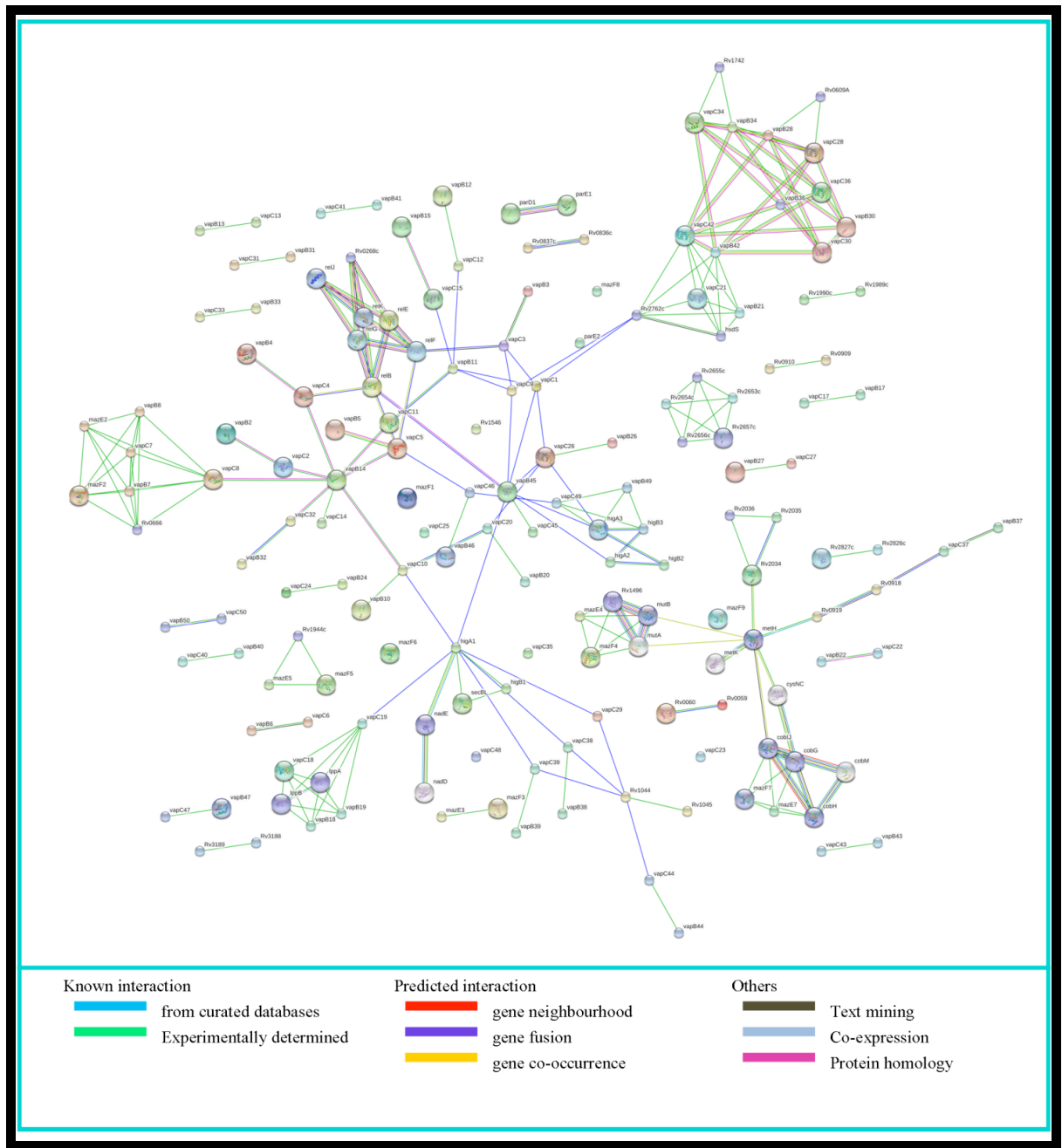

Figure 2: Protein-protein interaction network obtained and visualized by STRING v10.5 for input 79 Mtb TA systems. Nodes depict proteins and PPI are represented by edges in the network; interaction source of the PPI's are represented by various colors.

ISSN 0973-2063 (online) 0973-8894 (print)

Bioinformation 13(11): 380-387 (2017)
BIOMEDICAL

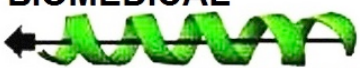




\section{BIOINFORMATION Discovery at the interf ace of physical and biological sciences}

\section{Open access}

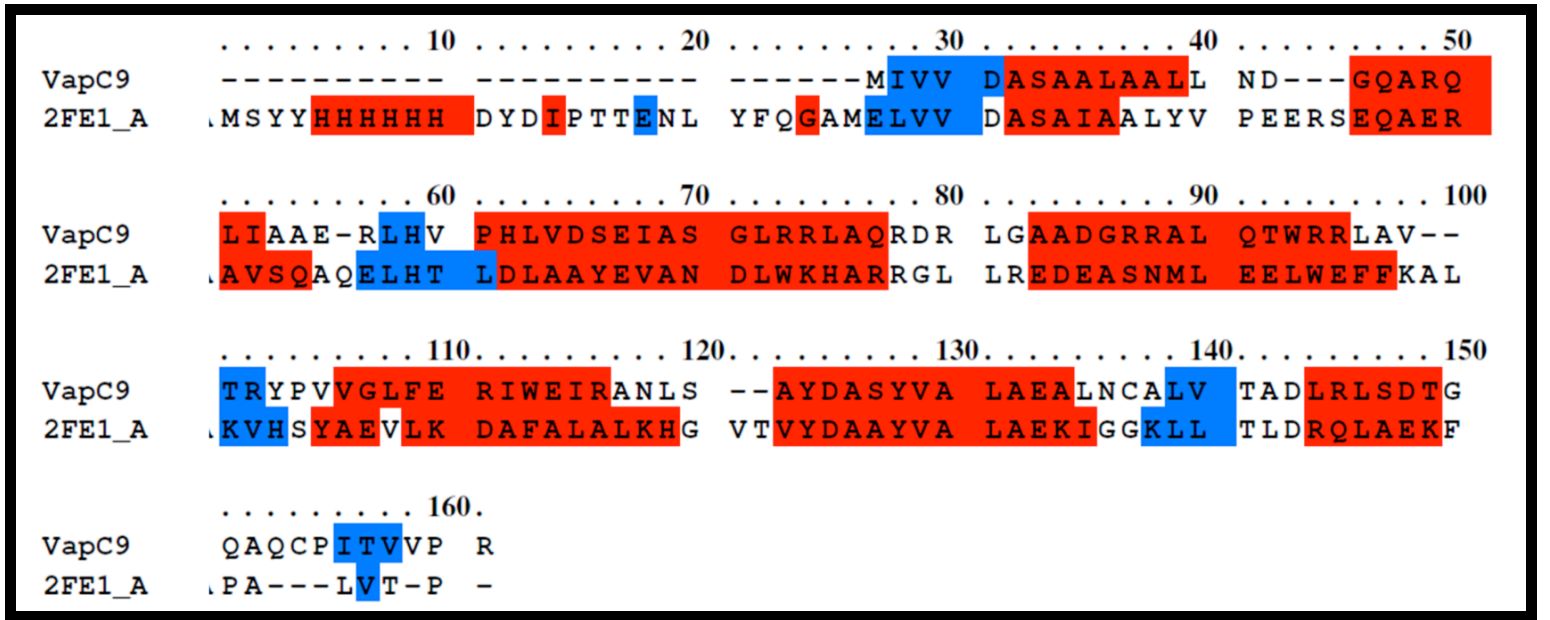

Figure 3: Sequence alignment of VapC9 with 2FE1_A obtained by PRALINE. Red color represents helix and blue color represents strand predicted by DSSP and PSIPRED.

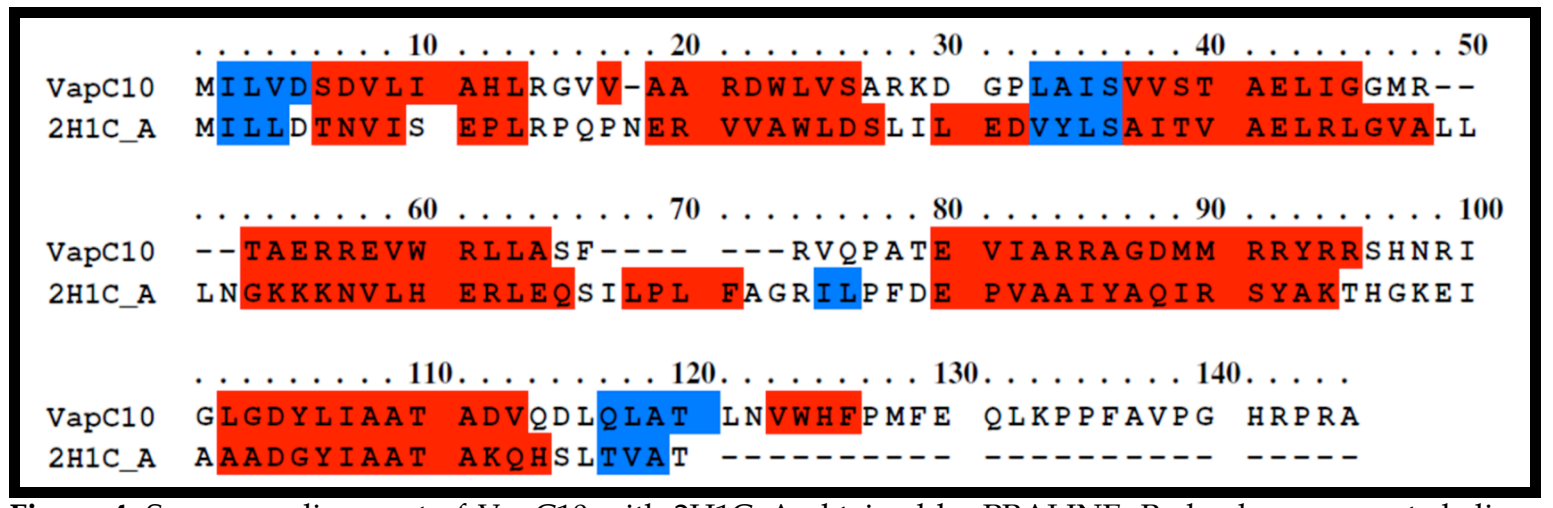

Figure 4: Sequence alignment of VapC10 with 2H1C_A obtained by PRALINE. Red color represents helix and blue color represents strand predicted by DSSP and PSIPRED.

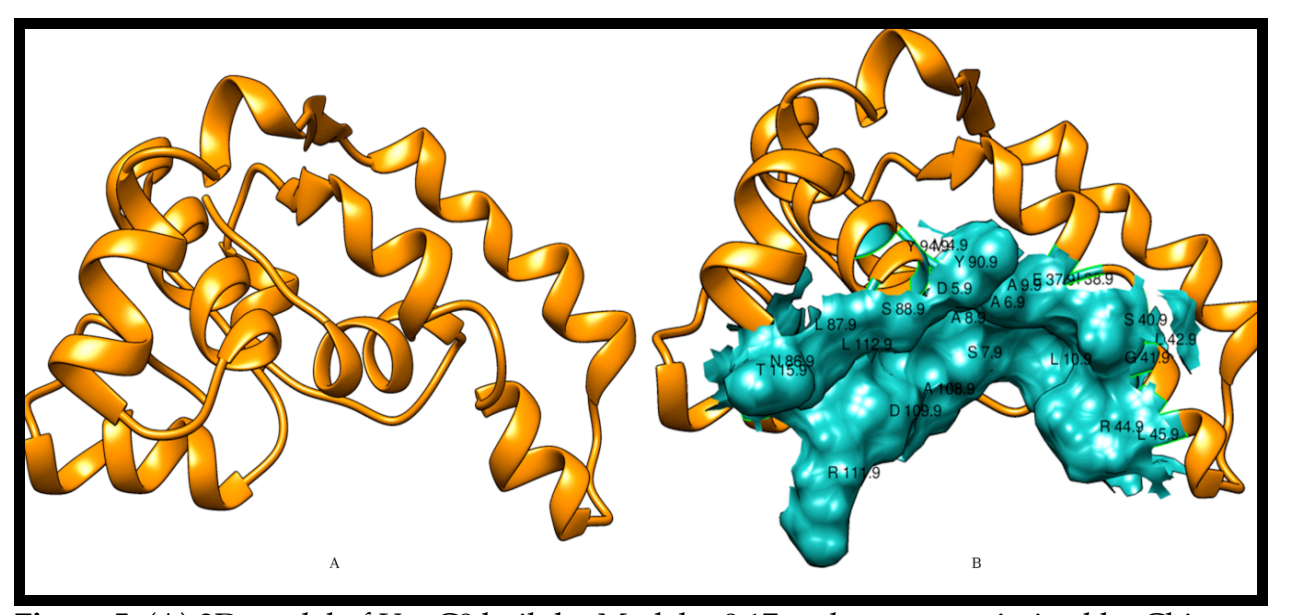

Figure 5: (A) 3D model of VapC9 built by Modeler 9.17 and energy optimized by Chimera v1.11.2. (B) Active site of VapC9 determined by Metapocket v2.0; active site residues are labeled with amino acid identifier and residue number.

ISSN 0973-2063 (online) 0973-8894 (print)

385

\section{BIOMEDICAL}

Bioinformation 13(11): 380-387 (2017)

(C)2017

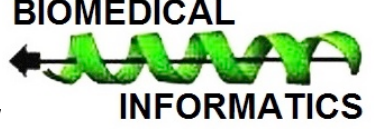




\section{BIOINFORMATION}

\section{Discovery at the interface of physical and biological sciences}

\section{Open access}

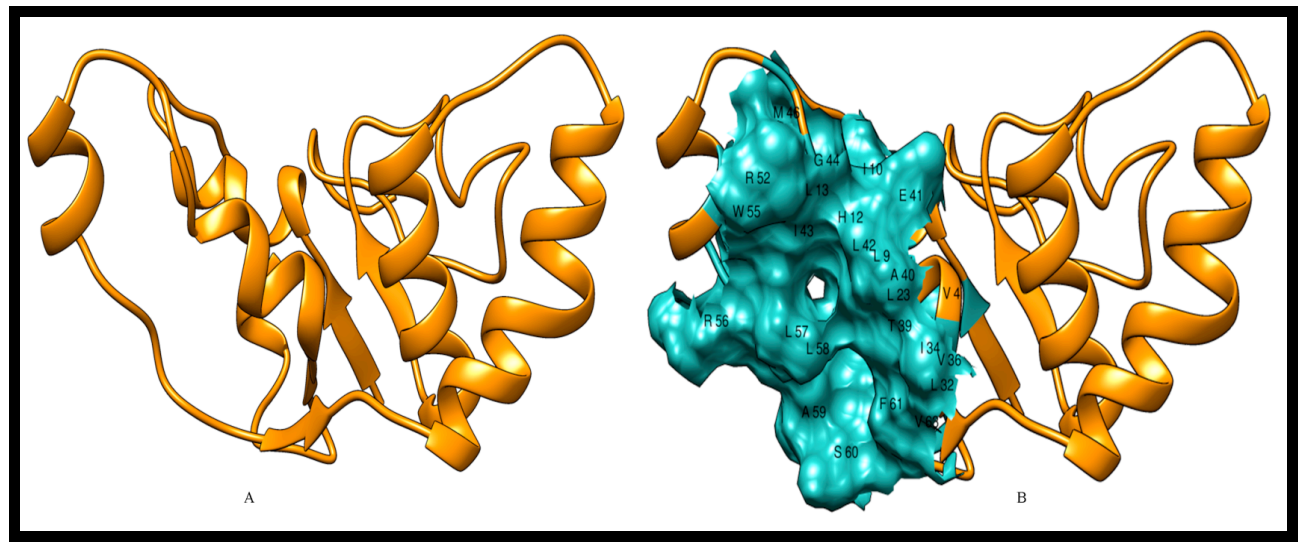

Figure 6: (A) 3D model of VapC10 constructed by Modeller 9.17 and energy optimized by Chimera v1.11.2. (B) Active site of VapC10 determined by Metapocket v2.0; active site residues are labeled with amino acid identifier and residue number.

Table 3: GO molecular function enrichment of PPI network for 79 MTb TA systems revealed over-representation of 11 GO ontology terms.

\begin{tabular}{llll}
\hline Pathway ID & Pathway description & Count in gene set & False discovery rate \\
\hline GO:0004518 & nuclease activity & 61 & $6.27 \mathrm{E}-62$ \\
GO:0004540 & ribonuclease activity & 49 & $7.72 \mathrm{E}-61$ \\
GO:0000287 & magnesium ion binding & 33 & $4.71 \mathrm{E}-17$ \\
GO:0046872 & metal ion binding & 48 & $7.36 \mathrm{E}-12$ \\
GO:0005488 & Binding & 74 & $1.39 \mathrm{E}-11$ \\
GO:0004519 & endonuclease activity & 15 & $7.51 \mathrm{E}-10$ \\
GO:0003677 & DNA binding & 24 & $3.91 \mathrm{E}-06$ \\
GO:0097351 & toxin-antitoxin pair type II binding & 5 & $5.88 \mathrm{E}-06$ \\
GO:0004521 & endoribonuclease activity & 6 & 0.000254 \\
GO:0003674 & molecular_function & 72 & 0.00188 \\
GO:0003676 & nucleic acid binding & 25 & 0.00196 \\
\hline
\end{tabular}

Table 4. List of top 15\% hub proteins identified in PPI network of Mtb TA systems by consensus of betweenness and radiality statistical measures.

\begin{tabular}{llllll}
\hline Name of protein & Rv number & Rank by & \multicolumn{3}{l}{ Score by } \\
\cline { 3 - 6 } higA1 & & Betweeness & Radiality & Betweeness & Radiality \\
vapB45 & Rv1956 & 1 & 2 & 2871.367 & 4.362833 \\
vapB14 & Rv2018 & 2 & 1 & 2762.824 & 4.446609 \\
Rv2762c & Rv1952 & 3 & 7 & 2189.49 & 4.201723 \\
vapC1 & Rv2762c & 4 & 15 & 2100.5 & 4.001948 \\
vapC9 & Rv0065 & 5 & 3 & 1528.757 & 4.337055 \\
vapB11 & Rv0960 & 6 & 4 & 1235.921 & 4.311278 \\
vapC10 & Rv1560 & 7 & 9 & 1181.667 & 4.092169 \\
relB & Rv1397c & 8 & 8 & 1096.531 & 4.175946 \\
vapC19 & Rv1247c & 9 & 6 & 1006.576 & 4.233945 \\
vapC5 & Rv2548 & 11 & 19 & 800 & 3.88595 \\
vapC11 & Rv0627 & 12 & 11 & 769.2976 & 4.047059 \\
higA3 & Rv1561 & 15 & 13 & 678 & 4.014837 \\
vapC26 & Rv3183 & 17 & 14 & 478.7214 & 4.008393 \\
vapC3 & Rv0582 & 18 & 5 & 452.619 & 4.253278 \\
vapC39 & Rv0549c & 19 & 12 & 407.0119 & 4.03417 \\
vapC38 & Rv2530c & 20 & 19 & 373.3333 & 3.88595 \\
relF & Rv2494 & 20 & 19 & 373.3333 & 3.88595 \\
vapC4 & Rv2865 & 22 & 22 & 347.5 & 3.873061 \\
& Rv0595c & 25 & 16 & 272.7 & 3.956838
\end{tabular}




\section{BIOINFORMATION}

\section{Discovery at the interface of physical and biological sciences}

\section{Open access}

Table 5: Physico-chemical properties of VapC9 and VapC10.

\begin{tabular}{lll}
\hline Physicochemical properties & VapC9 & VapC10 \\
\hline Theoretical pI & 13858.96 & 14952.43 \\
Molecular weight & 8.84 & 10.95 \\
Extinction coefficient & 15595 & 19480 \\
Instability index & 32.85 & 47.8 \\
Aliphatic index & 116.93 & 102.71 \\
Grand average of hydropathicity (GRAVY) & 0.15 & -0.008 \\
\hline
\end{tabular}

Table 6. Model evaluation scores of VapC9 and VapC10.

\begin{tabular}{|c|c|c|c|}
\hline Server & Parameter & VapC9 & VapC10 \\
\hline & Most favored regions (\%) & $93.00 \%$ & $84.50 \%$ \\
\hline & $\begin{array}{l}\text { Additionally allowed regions } \\
(\%)\end{array}$ & $6.10 \%$ & $12.90 \%$ \\
\hline & Generously allowed regions & & \\
\hline & & $0.90 \%$ & $2.60 \%$ \\
\hline & Disallowed regions (\%) & $0.00 \%$ & $0.00 \%$ \\
\hline \multirow[t]{2}{*}{ PROCHECK } & Overall G-factor (\%) & -0.32 & -0.27 \\
\hline & Z-score & -2.172 & -1.478 \\
\hline SWISS- & Q-Mean score & 0.543 & 0.609 \\
\hline MODEL & D-fire energy & -152.76 & -155.58 \\
\hline ERRAT & Overall quality (\%) & $97.30 \%$ & $93.50 \%$ \\
\hline \multirow{2}{*}{ ProSA-web } & Z score & -4.07 & -3.64 \\
\hline & LG score & 5.248 & 3.15 \\
\hline \multirow[t]{3}{*}{ ProQ } & MaxSub & 0.232 & 0.046 \\
\hline & $\mathrm{C} \beta$ deviations $>0.25 \mathrm{~A}^{\circ}(\%)$ & $0.00 \%$ & $0.00 \%$ \\
\hline & Residues with bad bonds (\%) & $0.00 \%$ & $0.00 \%$ \\
\hline MOLPROBITY & Residues with bad angles (\%) & $0.89 \%$ & $0.75 \%$ \\
\hline ResProx & Predicted Resolution $(\AA)$ & 1.375 & 1.964 \\
\hline
\end{tabular}

\section{Conclusion:}

We reported the construction and extensive analysis of PPI network of $79 \mathrm{Mtb}$ TA systems. Our computational analysis revealed significantly enriched gene ontology terms for pathways and molecular functions of $M t b$ TA systems and topological properties of PPI network. The major contribution is the identification of hub proteins of PPI network that can be explored as promising drug targets and for vaccine development. In addition, homology models of hub proteins VapC9 and VapC10 provide insights to its molecular functions.

\section{Acknowledgements:}

ZT acknowledges UGC-BSR for providing senior research fellowship. RD acknowledges MD University for providing University research scholarship. The work was supported by Department of Biotechnology (DBT), Govt. of India, New Delhi (BT/PR5641/MED/29/557/2012) and DBT-Bioinformatics Facility, MDU, Rohtak.
References:

[1] Lin W et al. 2016 Infect. Immun. 84:2505 [PMID: 27324481]

[2] Tiwari P et al. 2015 Nat. Commun. 6:6059 [PMID: 25608501]

[3] Sala A et al. 2014 Toxins (Basel). 6:1002 [PMID: 24662523]

[4] Kedzierska B \& Hayes F. Molecules. 2016 21:E790 [PMID: 27322231]

[5] Wang Y et al. J Proteome Res. 2010 9:6665 [PMID: 20973567]

[6] Nguyen CD et al. J. Biomed. Inform. 2011 44:824 [PMID: 21571095]

[7] Huo T et al. BMC Bioinformatics. 2015 16:100 [PMID: 25887594]

[8] Rao VS et al. Int J Proteomics. 2014 2014:147648 [PMID: 24693427]

[9] Szklarczyk D et al. Nucleic Acids Res. 2015 43:D447 [PMID: 25352553]

[10] Su Get al. Curr. Protoc. Bioinforma. 2014 [PMID: 25199793]

[11] Chin CH et al. BMC Syst. Biol. 2014 8:S11 [PMID: 25521941]

[12] Lew JM et al. Tuberculosis (Edinb). 2011. 91:1 [PMID: 20980199]

[13] Finn RD et al. Nucleic Acids Res. 2017 45:D190 [PMID: 27899635]

[14] Finn RD et al. Nucleic Acids Res. 2016 44:D279 [PMID: 26673716]

[15] Marchler-Bauer A et al. Nucleic Acids Res. 2017 45:D200 [PMID: 27899674]

[16] Wilkins MR et al. Methods Mol Biol. 2005 112:531 [PMID: 10027275]

[17] Arnold K et al. Bioinformatics. 2006 22:195 [PMID: 16301204]

[18] Colovos C \& Yeates TO. Protein Sci. 1993 2:1511 [PMID: 8401235]

[19] Wallner B \& Elofsson A. Protein Sci. 2003. 12:1073 [PMID: 12717029]

[20] Chen VB et al. Acta Crystallogr. Sect. D Biol. Crystallogr. 2010 66:12 [PMID: 20057044]

[21] Berjanskii M et al. J. Biomol. NMR. 2012 53:167 [PMID: 22678091]

[22] Wiederstein M \& M J Nucleic Acids Res. 2007 35:W407 [PMID: 17517781]

[23] Huang B. Omi. A J. Integr. Biol. 2009 13:325 [PMID: 19645590]

[24] Vallabhajosyula RR et al. PLoS One. 2009 4:e5344 [PMID: 19399170]

[25] Li Z et al. Nat. Commun. 2017 8:14356 [PMID: 28205554]

[26] Muetze T et al. F1000Research. 2016 5:1745[PMID: 27853512]

[27] Lin C. Y et al. Nucleic Acids Res. 2008 36:W438 [PMID: 18503085]

[28] Lee IG et al. Nucleic Acids Res. 2015 43:7624[PMID: 26150422]

[29] Arcus VL et al. Trends Microbiol. 2005 13:360 [PMID: 15993073]

Edited by $P$ Kangueane Citation: Thakur et al. Bioinformation 13(11) 380-387 (2017) License statement: This is an Open Access article which permits unrestricted use, distribution, and reproduction in any medium, provided the original work is properly credited. This is distributed under the terms of the Creative Commons Attribution License 